\title{
Effect of ELF Magnetic Stimulation on Distortion Product of Otoacoustic Emission in Tinnitus Patients
}

\author{
M. Pankowska ${ }^{a, *}$, D. Hojan-Jezierska ${ }^{a, b}$, E. SkrodzkA $^{c}$, E. SzYmieC $^{d}$, L. Kubisz $^{a, b}$, \\ T. ŚWIDZIŃSKI ${ }^{a}$ AND A. WICHER ${ }^{c}$ \\ ${ }^{a}$ Department of Biophysics, University of Medical Sciences, Poznań, Poland \\ ${ }^{b}$ Higher Vocational State School, Piła, Poland \\ ${ }^{c}$ Institute of Acoustics, A. Mickiewicz University, Poznań, Poland \\ ${ }^{d}$ ENT Department, University of Medical Sciences in Poznań, Poland
}

\begin{abstract}
One of the methods for objective evaluation of the hearing system is based on acoustic otoemissions. Particularly promising is the method based on measurement of the distortion product of otoacoustic emission as it is connected to the problem of tinnitus. Slow-varying magnetic field of low induction has been used in therapy of many diseases and ailments. It is expected that magnetostimulation will be effective in treatment of certain types of tinnitus by inducing the return of the organism to homeostasis. The aim of this study was to identify the changes in distortion product of otoacoustic emission levels in patients, prior and after the magnetostimulation. However any significant changes were found among patients with tinnitus, for the control group statistically important changes in distortion product of otoacoustic emission levels were observed.
\end{abstract}

PACS: 87.50.C-, 87.50.ct

\section{Introduction}

\subsection{Theory}

Magneto-stimulation and magnetotherapy make elements of physiotherapy based on the therapeutic effect of magnetic fields on human organism. Magnetotherapy performed with the use of permanent magnets has been known and applied for a few thousand years. The use of varying magnetic fields of different induction values and different shapes of the pulses is much more recent. According to a recent study, varying magnetic fields of low induction have been found effective in the therapy of many diseases and ailments [1-3]. One of many devices used for magneto-stimulation and magnetotherapy is the Viofor JPS System [4]. It involves exposition of the human body to magnetic field of extremely low frequencies and low magnetic induction (ELF_MF). The frequency of the basic course of the magnetic field ranges from several $\mathrm{Hz}$ to $3 \mathrm{kHz}$; its magnetic induction changes from $1 \mathrm{pT}$ to $100 \mu \mathrm{T}$. The envelope of the course is a wave whose frequency varies from several to $100 \mathrm{~Hz}$, while the frequency of the magnetic field used is lower than $100 \mathrm{~Hz}$ and its magnetic induction varies from $0.1 \mathrm{mT}$ to $20 \mathrm{mT}$. The biological effects of varying magnetic field generated by Viofor JPS System result from (a) electrodynamic effect on ion currents in the body, (b) magnetomechanic effect of the magnetic field of particles with uncompensated

* corresponding author; e-mail: mmat@amu.edu.pl magnetic spin and (c) ionic cyclotron resonance of ions in the body fluids. The main effects of biological magneto-stimulation mechanisms on the nervous system are analgesic, regenerative, vasodilative, anti-inflammatory, relaxing and antiseptic. For magnetotherapy, the two latter effects seem to be the most important.

The anti-inflammatory effect is related to the stimulation of formation of $3^{\prime}-5^{\prime}$-cyclic adenosine monophosphate (c-AMP) and E prostaglandin. Prostaglandin influences the accumulation of c-AMP, which decreases the secretion of inflammation mediators from neutrophiles, basophiles, masticates and lymphocytes. As to the effects on the nervous system, magnetic fields also improve the interneuron conductivity and modulate the neuron activity as well as the pineal gland's twenty-four hour rhythms of melatonin secretion. The pulsating magnetic fields generated by the Viofor JPS clearly modify the amplitude of the alpha and theta wave rhythms in proper directions, characteristic of the physiological state of relaxation. The magneto-stimulation by Viofor JPS system improves also blood circulation. It is particularly recommended to treat pains of various origins, motor system diseases or neurological diseases and has been very effective in posttraumatic, post-burn and neurological rehabilitation $[5,6]$.

In the search for possible methods of tackling the annoying problem of tinnitus, the idea of using slow-varying magnetic fields has appeared to improve the general condition of the hearing system and consequently to reduce or eliminate tinnitus of different aetiology. 


\subsection{Tinnitus}

Tinnitus is a conscious experience of sound that originates in the head, without any corresponding external source. Tinnitus noises are described as ringing, whistling, buzzing or humming. The noise may be continuous or it may come and go. There may be a single noise or two or more components. The noise may be heard in one ear, both ears or at the centre of the head. Sometimes, it may be difficult to pinpoint its exact location. The noise may be low, medium or high-pitched. Approximately $17 \%$ of the population in the world experiences persistent or troublesome tinnitus. Many auditory system structures participate in the mechanism of tinnitus generation. Nowadays, it is possible to identify the region responsible for tinnitus generation [7]. Zenner and Pfister [8] proposed three different tinnitus mechanisms connected with conductive, sensorineural and central tinnitus generation sites. Conductive tinnitus can be caused by some type of vibrations in the middle ear. Sensorineural tinnitus can be divided into several subtypes, including (a) "motor" tinnitus, connected with outer hair cells, (b) "transduction" tinnitus connected with inner hair cells, (c) "transformation" tinnitus generated from the auditory nerve and (d) "objective" tinnitus connected with extrasensory structures (vascular, muscular or other somatic sources).

The most prevalent explanations of the pathophysiological backgrounds of tinnitus generation mechanisms are connected with hair cells, the auditory nerve and central auditory nervous systems [9-12]. One of the possible mechanisms of generation of tinnitus of cochlear origin involves changes in the receptor potential of the hearing cells caused by damage to the ionic channels. Any disturbances in the ionic channel activity lead to changes in the receptor potential, which can lead to incorrect release of the neurotransmitter in the inner hair cells or disturb the control of movements of the outer hair cells. The theories claiming that the origin is related to the hair cell functioning indicate the following reasons of its defective functioning related to calcium imbalance, activations of NMDA ( $N$-methyl- $D$-aspartic acid) receptors, excitatory drift in hair cells and enhanced glutamate activity from the inner hair cells in response to stress. The theories claiming that the origin of tinnitus is related to the central auditory nerve system point to the effects of the efferent auditory system, increased spontaneous activity in the dorsal cochlear nucleus and generation of tinnitus by broad multimodal networks of neurons [13].

Many complementary and alternative therapies have been also proposed. It is worth mentioning acupuncture, aromatherapy, homeopathy or herbal medicine. Also, behavioural techniques are used to tame tinnitus. The tinnitus suppressive effect of electric stimulation and repetitive transcranial magnetic stimulation has been observed in some patients [14-17].

\subsection{Otoacoustic emission}

Objective evaluation of the hearing system can be performed with the use of tonal audiometry, auditory brain- stem response or acoustic otoemissions. Particularly promising is the method based on the distortion product of otoacoustic emission (DPOAE) as it is related to the phenomenon of tinnitus [18, 19].

The DPOAE is believed to arise from the nonlinear work of the cochlea stimulated by signals with frequencies $f_{1}$ and $f_{2}$. The generation of DPOAE is connected with the processes taking place in the cochlea responsible for the amplification of vibrations on the basilar membrane. Due to intermodulation, the cochlea generates a long series of components which are not present in the input stimuli. These components are called distortion products. The most prominent and frequently used in clinical practice is the cubic difference distortion product denoted as $2 f_{1}-f_{2}$. The level of amplitude for the frequency $2 f_{1}-f_{2}$ depends on the intensity and the ratio of its components with the frequencies $\left(f_{2} / f_{1}\right)$. For the human ear it lies in the range $0-10 \mathrm{~dB}$ SPL, in a comparatively wide range of frequencies. The components of DPOAE for the normal hearing are in the range of frequencies $0.9-10 \mathrm{kHz}$ and their maximum values lie in the band of $1-3 \mathrm{kHz}$.

The frequency of occurrence of DPOAE emission is 90-95\% for normal hearing population. According to the preliminary results of the acoustic otoemission study in healthy subjects, magneto-stimulation is correlated with increasing amplitudes of DPOAE [20].

Some authors studying DPOAE in tinnitus patients claim that DPOAE levels decrease in tinnitus ears with clinically normal hearing when compared to the DPOAE levels in normal hearing (nontinnitus) ears [21]. However, according to other authors, DPOAE levels in tinnitus ears may be lower or higher, depending on the frequency range $[22,23]$. Thus, the results are ambiguous.

In the present study, attempts are made to determine the impact of magnetostimulation with strictly defined parameters on the level of DPOAE in patients with tinnitus.

\section{Experimental procedure}

The study on magnetostimulation on the human body was approved by the local Ethical Committee.

The group of 5 normally hearing persons (normal) and 11 tinnitus patients (tinnitus) participated in the preliminary studies of magnetostimulation effects on auditory system. The age range was within $25-58$ years for healthy and 46-72 for tinnitus subjects. Tinnitus patients were selected from those treated at the Center of Laryngological Rehabilitation Medical University in Poznań. All participants expressed written formal consent to take part in the study. In order to estimate their wellness and possible contraindications to participate in the experiment, the subjects were examined by means of a specific questionnaire. Prior to magnetostimulation patients were examined using a tonal audiometer. Next the tinnitus pitch and tinnitus loudness were measured. In our experiment the tinnitus matching stimuli were presented to the ipsilateral ear using the same audiometer as in the hearing threshold measurements, with the subject posi- 
tioned in a sound-proof booth. Depending on the sensation of the tinnitus pitch, pure tones or narrow band-pass noises were used as matching stimuli. They were presented at level corresponding to the tinnitus loudness. Furthermore, the DPOAE audiograms (DP-grams) were recorded in soundproof room by Audera Grason-Stadler system. Primary tones were presented at the level of $L_{1}=65 \mathrm{~dB}$ SPL and $L_{2}=55 \mathrm{~dB}$ SPL. The $f_{2} / f_{1}$ ratio was set on 1.22. The responses were measured at $2 f_{2}-f_{1}$ with respect to $f_{2}$. The DP-grams were collected in the $f_{2}$ frequency range from 1 to $8 \mathrm{kHz}$ and they were accepted as valid when the signal-to-noise ratios of the $2 f_{1}-f_{2}$ component exceeded $6 \mathrm{~dB}$.

The intensity level of primary tones was held constant at all frequencies tested and DPOAE data were plotted for different frequencies. After the magnetostimulation, the patients' DP-grams were measured again to determine changes in DPOAE's amplitudes. The magnetostimulation was carried out using the Viofor JPS System with $65 \mathrm{~cm}$ ring-shaped applicator.

Topography of the magnetic field was determined by the hallotronic probe, which had been calibrated previously. Prior to the determination of the magnetic field topography of the coil, a regular net built of $1 \times 1 \mathrm{~cm}^{2}$ squares was constructed. The measurements were performed at the front plane of the applicator. Magnetic field was generated in the continuous mode. The magnetic induction and the course of the magnetic field was recorded using a C.A42 magnetometer (Chauvin Arnoux) equipped with the external isotropic sensing hallotron element MF05, which allowed measurements of the three components: $B_{x}, B_{y}, B_{z}$ to the accuracy of $10 \mu \mathrm{T}$. The recorded data were supplied to a computer for further analysis. The measurements were carried out in the centre of the applicator.

The obtained topography of the magnetic field showed that the magnetic field was relatively homogeneous with smaller magnetic induction in the central part of the plane of measurements (Fig. 1). Furthermore, it was surrounded by a ring of $20 \%$ higher magnetic induction. In the vicinity of the wall of the applicator, the field was irregular and its intensity values differed from those in the centre. The courses of magnetic field used in the experiment are shown in Figs. 2 and 3.

Viofor JPS offers the possibility of magnetic field application in two programs M2P2 and M1P3. The first program was presented to the subject at a constant intensity of 6 (default value of the apparatus) and then, after $20 \mathrm{~min}$ break, the subject was exposed to the second program presented at the intensity of 5 . Parameters of the magnetic field used in magnetostimulation and magnetotherapy are crucial for the biological effects produced in the exposed tissue. Usually it is not possible to examine the actual shape and size of the course of the magnetic field. The courses of the magnetic field determined in the experiment were complex. Figures 2 and 3 show the $250 \mathrm{~s}$ long fragments of the recorded courses. Generally, for any programme, which is available in the

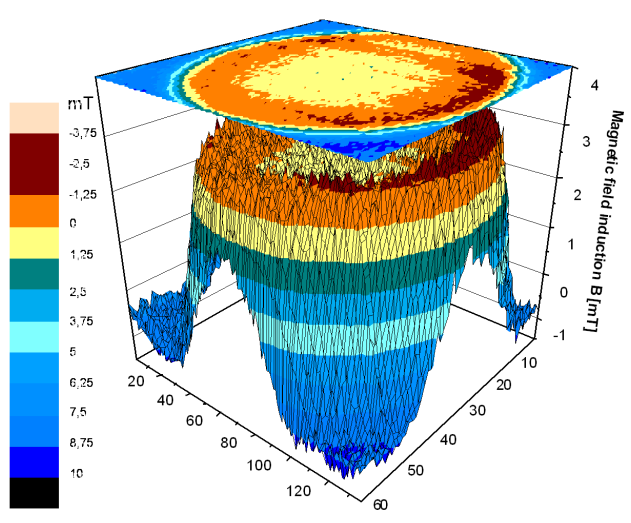

Fig. 1. Magnetic field distribution in the $z$-axis, generated by the $65 \mathrm{~cm}$ ring-shaped applicator.

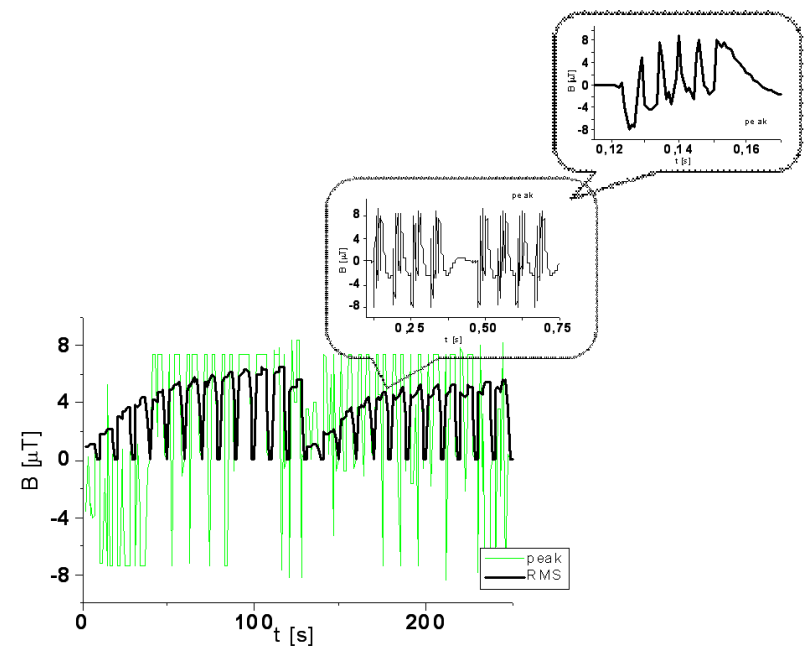

Fig. 2. Hierarchical structure of the magnetic field course P3M2 (z-component).

Viofor JPS, the envelope of the root mean squared (RMS) values contains shorter packets. Each packet is formed by a series of much shorter pulses of different shapes. Individual signals are first grouped in pockets, and then in series and sets. They are sent as various combinations in appropriate application programmes, described as M1, M2 etc. The time scale for these three components is as follows: approximately $120 \mathrm{~s}$ for the envelope forming a series, $0.25 \mathrm{~s}$ for the packet and about $30 \mathrm{~ms}$ for individual impulses.

Each treatment session is described by the following parameters: program $(P)$, application mode $(M)$ and intensity level $(I) . P 1$ program consists of one type of pulses, $P 2$ of two types of pulses and $P 3$ of two types of pulses, with a different sequence than in $P 2$.

$M 1$ stands for exposure to the magnetic field of a constant intensity and this mode of presentation is recommended for subjects of good general health condition. M2 stands for exposure to the magnetic field of increasing intensity, which is recommended for subjects of poor 


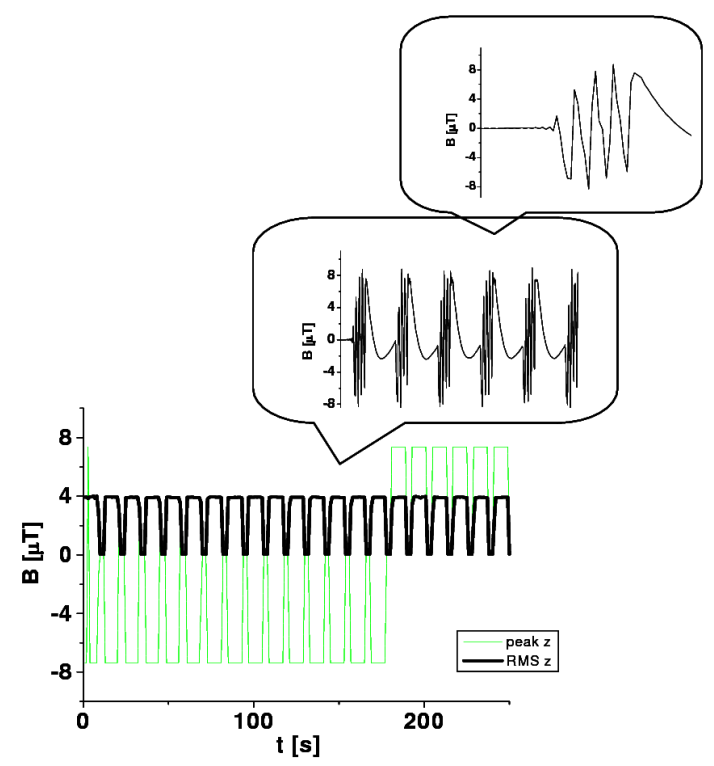

Fig. 3. Hierarchical structure of the magnetic field course P2M1 (z-component).

general health condition. M3 stands for exposure to the magnetic field of increasing and then decreasing intensity.

\section{Results and conclusions}

For the control group (normal) the hearing threshold did not exceed $20 \mathrm{~dB}$ HL. For the group of tinnitus patients (tinnitus) the audiograms show that all patients exhibited sensorineural hearing losses characterized by a sloping audiogram for higher frequencies.

The tinnitus appeared as a ton-like sensation at the frequency range $4-8 \mathrm{kHz}$ in almost $80 \%$ cases and in the remaining cases as a noiselike sensation with a central frequency of a noise band in the frequency range 200-500 Hz. To evaluate the influence of magnetostimulation on the DPOAE level for the control group (normal), the 3 factor variance analysis was performed. The factors " $f_{2}$ frequency" $\{F(9,79)=21.110, p=0.001\}$ and "normal" $\{F(4,79)=19.705, p=0.001\}$ were found to significantly influence the DPOAE level, however the influence of the factor "magnetostimulation" $\{F(1,79)=$ 10.559, $p=0.002\}$ was significant. The couplings of the factors " $f_{2}$ frequency" and "magnetostimulation" were statistically insignificant (Fig. 4) but "normal" and "magnetostimulation" were statistically significant (Fig. 5) and that the mean DPOAE level was increased after magnetostimulation.

The DPOAE levels, before and after the procedure of magnetostimulation, were also compared for the group of tinnitus patients and hearing loss sufferers (tinnitus). The 3 factor variance analysis showed that the factor "magnetostimulation" was statistically insignificant $\{F(1,220)=0.345, p=0.557\}$. The mean differences in DPOAE levels for individual patients (tinnitus) were much different; for some patients (tinnitus) the DPOAE level was decreased after magnetostimulation, while for others this level increased. Figure 6 presents the effect of coupling of the factors "tinnitus" and "magnetostimulation", which was shown to be statistically significant $\{F(10,220)=2.255, p=0.0158\}$.

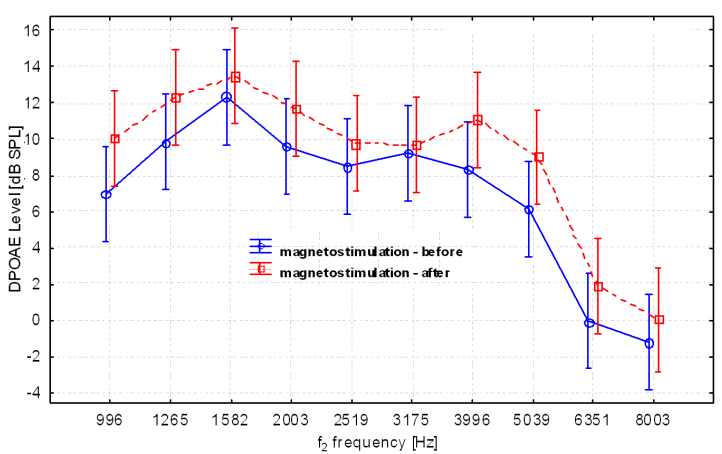

Fig. 4. The coupling of the factors " $f_{2}$ frequency" and "magnetostimulation" for the control group (normal).

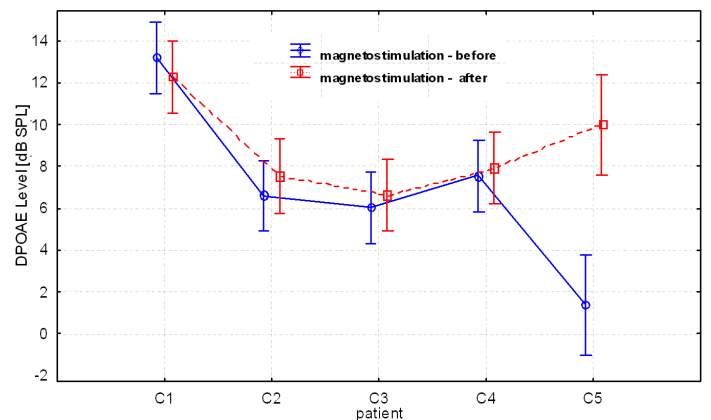

Fig. 5. The coupling of the factors "the control group (normal)" and "magnetostimulation".

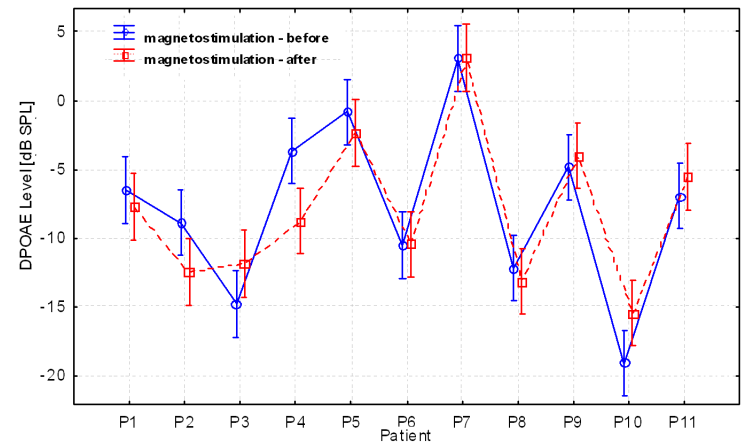

Fig. 6. The coupling of the factors "tinnitus" and "magnetostimulation".

On the basis of the above presented results we are able to draw the following conclusions:

1. Statistically significant influence of ELF magnetostimulation on the level of DPOAE amplitudes 
has been observed in persons of normal hearing without tinnitus.

2. In persons with hearing loss and tinnitus the influence of ELF magnetostimulation on the level of DPOAE amplitudes has not been noted.

3. Results of this study can be the starting point of possible therapeutic indications of tinnitus treatment by ELF type magnetostimulation.

In order to identify the direction of DPOAE amplitude changes as a result of magnetostimulation it is necessary to analyse in more detail the origins and types of hearing loss and tinnitus in the persons studied.

\section{References}

[1] A. Sieron, Practical Use of the Electromagnetic Fields in Medicine, 2nd ed., $\alpha$-medica press, Bielsko-Biała 2002 (in Polish).

[2] M. Pecyna, Psychophysiological Perspective on Low-Field Magnetic Induction, Medsport, Warszawa 2004 (in Polish).

[3] J. Pasek, R. Mucha, A. Sieroń, Fizjoterapia 14, 3 (2006).

[4] M.P. Dąbrowski, Pediatr. Med. Rodz. 1, 78 (2005) (in Polish).

[5] J. Pasek, A. Misiak, R. Mucha, T. Pasek, A. Sieroń, Fizjoterapia Polska 1, 8, 1 (2008) (in Polish).

[6] J. Pasek, T. Pasek, A. Sieroń, Acta Bio-Optica Informat. Med. 14, 284 (2007) (in Polish).

[7] J.A. Henry, K.C. Dennis, M.A. Schechter, J. Speech Language Hearing Res. 48, 1204 (2005).

[8] H.P. Zenner, M. Pfister, in: Proc. Sixth Int. Tinnitus Seminar, Ed. J. Hazell, London, The Tinnitus and Hyperacusis Centre, London 1999, p. 17.
[9] P.J. Jastreboff, Neurosci. Res. 8, 221 (1990).

[10] L.E. Roberts, J.J. Eggermont, D.M. Caspary, S.E. Shore, J.R. Melcher, J.A. Kaltenbach, J. Neurosci. 10, 30, 14972 (2010).

[11] E. Szymiec, Przew. Lek. 10, 99 (2002) (in Polish).

[12] M. Śliwińska-Kowalska, Clinical Audiology, 1st ed., Mediton, Łódź 2005 (in Polish).

[13] J.R. Melcher, I.S. Sigalovsky, J.J. Guinan, R.A. Levine, J. Neurophysiology 83, 1058 (2000).

[14] N.J. Roland, J.B. Hughes, M.B. Daley, J.A. Cook, A.S. Jones, M.S. McCormick, Clinical Otolaryngology Apllied Sci. 18, 278 (1993).

[15] R.L. Steenerson, G.W. Cronin, Otolaryngology Head Neck Surgery 121, 511 (1999).

[16] B. Langguth, G. Hajak, T. Kelinjung, S. Pridmore, P. Sand, P. Eichhammer, Acta Otolaryngol. Supp. 126, 102 (2006).

[17] E. Szymiec, W. Szyfter, M. Karlik, Otolaryngol. Pol. 51, 487 (1997).

[18] E. Ozimek, Postępy w Chirurgii Głowy i Szyi 1, 5 (2005) (in Polish).

[19] Y. Shiomi, J. Tsuji, Y. Naito, N. Fujiki, N. Yamamoto, Hearing Res. 108, 83 (1997).

[20] M. Pankowska, D. Hojan-Jezierska, E. Szymiec, in: VI Symp. on Medical Physics IV Int. Symp. on Medical Physics, Szczyrk (Poland), 2009, Abstracts, p. 76 (in Polish).

[21] E. Ozimek, A. Wicher, W. Szyfter, E. Szymiec, J. Acoust. Soc. Am. 119, 527 (2006).

[22] S.J. Norton, A.R. Schmidt, L.J. Stover, Ear Hear. 11, 159 (1990).

[23] C. Mitchell, D.J. Lilly, J. Henry, in: Proc. 5th Int. Tinnitus Seminar, Eds. G. Reich, J. Vernon, Am. Tinnitus Association, Portland 1995, p. 180. 